\title{
A Top-k Analysis Using Multi-level Association Rule Mining for Autism Treatments
}

\author{
Kelley M. Engle and Roy Rada \\ Department of Information Systems, \\ University of Maryland Baltimore County (UMBC), \\ Baltimore, MD 21250 USA \\ $\{$ kelley.engle, rada\} @umbc. edu
}

\begin{abstract}
Association rule mining is based on associations of attribute values in a database. To facilitate finding meaningful rules, we segment the database by a categorization of database records based on a taxonomy on one of the attribute value sets. To test the value of this approach we have applied it to a large database about treatment impacts on autistic children. The segmented analyses lead to interestingly, different results from the analyses done without segmentation.
\end{abstract}

Keywords: association rule mining, autism, data mining.

\section{Introduction}

Medical data analysis is challenging due to the inherently complex and critical nature of the data and the far reaching implications of the analysis. In this paper we propose a novel approach to combine domain knowledge with an association rule mining technique to generate association rules. Our application domain is treatments for autism.

\subsection{Data Mining Rules}

Association rules identify collections of data attributes that are statistically related in the underlying data. An association rule is of the form $\mathrm{X} \Rightarrow \mathrm{Y}$ where $\mathrm{X}$ and $\mathrm{Y}$ are disjoint conjunctions of attribute-value pairs. To illustrate, imagine a set of attributes \{milk,bread,butter,beer\}. The data comes from a grocery where each customer is represented by a conjunction of bought or not bought values for each of that attributes. If customers who buy bread always also buy butter, then one association rule could be 'bread is bought' $=>$ 'butter is bought'.

The confidence of the rule is the conditional probability of $\mathrm{Y}$ given $\mathrm{X}, \operatorname{Pr}(\mathrm{Y} \mid \mathrm{X})$, and the support of the rule is the prior probability of $\mathrm{X}$ and $\mathrm{Y}, \operatorname{Pr}(\mathrm{X}$ and $\mathrm{Y})$ where probability is the observed frequency in the data. The traditional association rule mining problem is:

- Given a database of transactions, a minimal confidence threshold and a minimal support threshold, 
- find all association rules whose confidence and support are above the corresponding thresholds.

Rules that meet the minimum support and confidence thresholds may be further analyzed for their correlation values. The correlation for $\{\mathrm{X}, \mathrm{Y}\}$ is computed as the support value for that pair, divided by the square root of the product of the support values for $\mathrm{X}$ and $\mathrm{Y}$. Rules that have a correlation value that is greater than some minimum correlation value will be retained.

Often in association rule mining it can be difficult to generate rules due to the sparsity of the data at the atomic level [1]. Multi-level association rule mining alleviates this problem by segmenting the data according to a user-defined hierarchy. Such segmenting will be performed in this research.

\subsection{Autism}

Autism is a neurodevelopmental disorder that appears during childhood and generally follows a steady course without remission. The characteristic triad of symptoms involves impairments in social interaction; impairments in communication; and restricted interests and repetitive behavior. Numerous disorders overlap in their symptomatology with those of autism, and the terminology and diagnosis in this area is often clouded but incidence can be broadly stated to be several children per thousand children.

For autistic children, no single treatment is best, and many, different approaches are often tried with each child. On average, an autistic child is on seven different treatments at any given point in time [2]. The efficacy of the majority of these treatments is not supported by clinical trials with few exceptions. In terms of available pharmacological agents, only risperidone and Ritalin have replicable results for the autistic population [3].

The Interactive Autism Network (IAN) web project is designed to serve as a national registry for autistic children and their families. Its purpose is two-fold: (1) to connect researchers to large, diverse subject bank and; (2) to collect data for secondary analysis and release the de-identified data to researchers. The IAN web project has registered thousands of families. There are more than 500 treatments in the IAN dataset. These treatments include medications, vitamin and mineral supplements, diets, behavioral/educational interventions as well as alternative treatments designed to treat the autistic child holistically. It would not be feasible or prudent to conduct clinical trials on such a large number of treatments. Data mining, for this particular scenario, would be helpful in reducing the solution space to determine the best treatments. The efficacy for this smaller group of treatments could then be properly confirmed in a clinical trial. Multiple analyses would be advantageous in pruning the solution space in order to overcome any limitations in one particular method.

\subsection{Autism and Data Mining}

The vast majority of autism research is conducted using "traditional" analysis methods such as statistics. There are few studies that have incorporated data mining methods for the autism domain. Because the current knowledge regarding autism is 
limited, borrowing methods from data mining could be a valuable aid in theorybuilding. In the following paragraphs, the few studies that have been conducted using methods from the data mining area are presented.

Self-organizing maps were used to model attention shift impairment and familiarity preference, both hallmarks of autistic behavior [4]. This work concluded that the familiarity preference exhibited by autistic patients may be in fact the primary cause of other autistic characteristics such as shift impairment.

Heart rate patterns were compared with behavioral problems such as self-injury and aggression which are common in the autistic population [5]. The analysis was conducted using LERS (Learning from Examples using Rough Sets) that generates classification rules from the presented data. The learning mechanism in LERS is similar to the bucket brigade algorithm [6, 7]. The analyses indicate that both high and very high heart rates are associated with problem behaviors. Further research avenues were discussed and included physiological data with behavioral treatment plans. The analyses of EEG data to support research in epileptology and autism was also presented [8].

An expert system was developed as a screener for developmental disorders including autism [9]. The purpose of the system was to enable clinicians in countries where specialists, such as developmental pediatricians, are few in number, diagnose autism and other developmental disorders in children at an earlier age. Text mining was applied to biomedical literature on autism in order to "identify potential contributions to a better understanding of autism [10]." The results of the text mining allowed for the construction of an ontology which identified "rare relations" in the autism data. This research led to the development of RaJoLink [11, 12], a literature mining method for "uncovering hidden relations from a set of articles in a given domain."

Genetic and environmental factors were examined using CFA (Combinatorial Fusion Analysis) and association rule mining to determine associations between autism prevalence and the exposure to mercury and lead during critical stages of a child development [13]. The results of these analyses indicate an association between an increase of mercury concentration in the environment with an increase in autism prevalence three years later.

\section{Method}

We use an association rule mining tool called Weka (www.weka.org) to implement a novel approach to rule mining. Our approach utilizes an external treatment ontology and multi-level association rules. We discuss detailed experimental results demonstrating the efficacy of our framework. Utilizing a host of measures including: (1) support, (2) confidence, (3) lift, (4) leverage, (5) conviction and, (6) the chi-square test, we were able to prune the original rules generated to include only those with strong positive correlation.

Our approach has two components:

- Association rule mining conducted on the entire treatment dataset and;

- Multi-level association rule mining based on a dataset segmented according to the treatment ontology. 
Furthermore, the multi-level association rule mining was conducted at two sub-levels:

- First an analysis based on only categories and not the individual treatments was conducted in order to find the categories most associated with improvement; and

- Second an analysis for each category was conducted which included the treatments in order to find the top-performing treatments.

In addition to the more commonly used support and confidence, we incorporated lift, leverage and conviction measures in our analyses in order to detect negative correlation in the rules. Additionally, we conducted a separate chi-square analysis in order to confirm the correlation as well as determine the strength of that correlation. Table 1 explains the interpretation of these measures.

Table 1. Explanation of measures

\begin{tabular}{ll}
\hline Measure & Interpretation \\
\hline Support & Minimum support was varied from .1 to .01 in order to pick \\
& up less commonly used treatments in the IAN dataset. \\
& Confidence was set very low .1 in order to pick up as many \\
& rules as possible. \\
& $<1-$ Negative correlation \\
& $=1-$ Independent \\
& $>1-$ Positive correlation \\
& The higher this value, the more likely that the rule is not just \\
& a random occurrence, but because of some relationship \\
& between them. \\
& $<0-$ Negative correlation \\
& $=0-$ Independent \\
& $>0-$ Positive correlation \\
& $<1-$ Negative correlation \\
& $=1-$ Independent \\
Leverage & $>1-$ Positive correlation \\
& Unlike lift, this measure is not symmetric which means it has \\
no upper bound. & The number itself denotes the strength of the correlation \\
& whereas a second heading is necessary to relate the direction \\
& of the correlation.
\end{tabular}

Finally we take all the rules generated using our ensemble methods and perform a top-K analysis on them to find the strong association rules. We applied this framework on the IAN data to identify strong association rules between treatments for autism and the outcomes of the treatments with promising results.

We collected data from IAN on 3,283 children In total, there were 14,351 treatment records with corresponding efficacy ratings.

There were three attributes used for this analysis: (1) Treatment name; (2) Treatment efficacy and; (3) Taxonomy. The treatment efficacy is rated by the parent using a 9-point Likert scale with four ratings for worsening, one for no change and four for improvement. During the course of our analysis, we found that consolidating 
the Likert scale was advantageous to the analysis particularly to garner enough support/confidence to produce meaningful rules.

The ontology used was borrowed from the IAN website where a number of categories were presented to the users (parents of autistic children) as they would search for and enter treatment information. The categories for the taxonomy include: (1) Complementary and Alternative; (2) Educational and Behavioral; (3) Medications; (4) Physiological; (5) Special Diets and; (6) Vitamins and Supplements and (7) Top Ten.

\section{Results}

The top three treatments from the original dataset were not necessarily surprising since they are widely available for most autistic children in various settings (home, community, and school). The results of the statistical measures including chi-square emphatically confirm that Applied Behavioral Analysis and Speech and Language Therapy, and Occupational Therapy are indeed positively correlated with improvement.

When analyzing by ontology - three categories seem to have the strongest positive correlation with improvement - these include Educational and Behavioral, Top Ten, and Medications. Top Ten is simply a category IAN used to compile the most commonly used treatments. Intuitively, associating the Top Ten category with improvement would seem judicious since most parents would probably not use treatments in such high numbers unless some improvement was noted.

In the Complementary and Alternative segment, 5 treatments had been associated with improvement. After making a segment specific analysis, only Prayer is considered a strong rule with a lift value of 1.19 , conviction of 2.55 and a very strong positive correlation. The rest of the treatments are considered weak rules with lift values below 0 (negative correlation), leverage values at 0 or below and conviction values below 1 (see Table 2).

Table 2. Complementary and Alternative

\begin{tabular}{|l|r|l|r|r|r|l|}
\hline Treatment & Confidence & Lift & Leverage & Conviction & $\mathbf{X}^{\mathbf{2}}$ & Correlation \\
\hline Prayer & 0.92 & 1.19 & 0.02 & 2.55 & 268.71 & Positive \\
\hline Weighted Blanket & 0.74 & 0.96 & -0.01 & 0.88 & 1.18 & Negative \\
\hline Chelation & 0.74 & 0.96 & 0 & 0.84 & 0.5 & Independent \\
\hline Music Therapy & 0.73 & 0.95 & -0.01 & 0.99 & 0.5 & Independent \\
\hline Equine Therapy & 0.72 & 0.93 & -0.01 & 0.8 & 2.1 & Negative \\
\hline
\end{tabular}

Doing a segment only analysis of medications also provided interesting results. Clonidine was confirmed as a positively correlated rule. Risperidone, on the other hand, had a lift measure of .99 and conviction of .95 which suggests a negative correlation and a chi-square value indicating independence. This is quite surprising since risperidone is one medication for autism that has shown efficacy in clinical trials [14].

The following criterion was used to include treatments in the top '10':

- Confidence $>.70$

- Two of the three statistical measures (lift, leverage and conviction) had to indicate 
positive correlation. For lift and conviction this meant for any value above 1 and for leverage any value above 0 .

- A positive correlation for the chi-square test.

This analysis of the top-10 does not indicate treatment efficacy, since that can only be determined in a properly controlled clinical study. However, out of the 500 treatments present in the IAN dataset, this top-10 list indicates those treatments most like to be effectual and good candidates for clinical study.

\section{Conclusion}

Multi-level association rule mining is an effective tool in allowing domain knowledge to guide model development. Through the use of a treatment ontology, we were able to extract more meaningful rules from the IAN dataset. Subsequent analyses of measures such as lift, leverage and a separate chi-square test pruned the list of treatments to include only those that are positively correlated. As a result, a top-k list of treatments for autism was presented.

Without segmentation, the only treatments shown to be effective were ABA (Applied Behaviorial Analysis), Speech and Language therapy and Occupational therapy. These three therapies are one of the most common treatments for autism and therefore had the support and confidence necessary to surface as rules due to the large number of autistic children receiving them. Segmenting the dataset via the treatment ontology provided an opportunity to examine alternate treatments that are not as widely used among the autistic population. Determining absolute efficacy of these treatments is not feasible using association rule mining. The results reported only indicate treatments that are good candidates for clinical studies.

The methodology presented could be extended to other medical domains that contain well-established ontologies. The inclusion of such ontologies can serve as a powerful aid in guiding the association rule mining in generating more significant rules. The inclusion of the other measures to determine correlation are an important part of the pruning process. Medical domains where a fundamental understanding of a particular disorder is limited and a rich dataset of patient data is available would benefit from this 'theory-building' approach.

\section{References}

1. Han, J., Kamber, M.: Data Mining: Concepts and Techniques, 2nd edn. Morgan Kaufman, Amsterdam (2006)

2. Green, V.A., et al.: Internet survey of treatments used by parents of children with autism. Research in Developmental Disabilities 27(1), 70-84 (2006)

3. Parikh, M.S., Kolevzon, A., Hollander, E.: Psychopharmacology of aggression in children and adolescents with autism: a critical review of efficacy and tolerability. Journal of Child and Adolescent Psychopharmacology 18(2), 157-178 (2008)

4. Paplinski, A.P., Gustafsson, L.: An attempt in modelling early intervention in autism using neural networks. In: 2004 IEEE International Joint Conference on Neural Networks, 2002. IEEE, Piscataway (2004) 
5. Freeman, R.L., Grzymala-Busse, J.W., Harvey, M.: Functional behavioral assessment using the LERS data mining system - Strategies for understanding complex physiological and behavioral patterns. Journal of Intelligent Information Systems 21(2), 173-181 (2003)

6. Booker, L.B., Goldberg, D.E., Holland, J.F.: Classifier systems and genetic algorithms. In: Carbonell, J.G. (ed.) Machine Learning Paradigms and Methods, pp. 236-282. Elsevier North-Holland, Inc., New York (1990)

7. Holland, J.H., Holyoak, K.J., Nisbett, R.E.: Induction processes of infererence, learning, and discovery. The MIT Press, Cambridge (1986)

8. Hrncir, Z., Komarek, V.: Analyses of EEG recordings. Neural Network World 14(1), 21-25 (2004)

9. Veeraraghavan, S., Srinivasan, K., Latifi, S.: Exploration of autism using expert systems. In: 4th International Conference on Information Technology New Generations. IEEE Comput. Soc., Los Alamitos (2007)

10. Petric, I., Urbancic, T., Cestnik, B.: Discovering hidden knowledge from biomedical literature. Informatica Slovenia 31(1), 15-20 (2007)

11. Urbancic, T., Cestnik, B., Macedoni-Luksic, M.: Literature mining method RaJoLink for uncovering relations between biomedical concepts. Journal of Biomedical Informatics 42(2), 219-227 (2009)

12. Urbancic, T., et al.: Literature mining: Towards better understanding of autism. In: Bellazzi, R., Abu-Hanna, A., Hunter, J. (eds.) AIME 2007. LNCS (LNAI), vol. 4594, pp. 217-226. Springer, Heidelberg (2007)

13. Schweikert, C., et al.: Analysis of autism prevalence and neurotoxins using combinatorial fusion and association rule mining. In: 9th IEEE International Conference on Bioinformatics and BioEngineering (BIBE), p. 400-404 (2009)

14. Aman, M.G., Arnold, L.E., Ramadan, Y.: Randomized, controlled, crossover trial of methylphenidate in pervasive developmental disorders with hyperactivity. Archives of General Psychiatry 62(11), 1266-1274 (2005) 